Supporting Information for

\title{
Mechanical forces have a range of effects on the rate of ribosome catalyzed peptidyl transfer depending on direction
}

Yang Jiang ${ }^{1}$ and Edward P. O'Brien ${ }^{1,2,3, *}$

1 Department of Chemistry, Penn State University, University Park, Pennsylvania, United States

2 Bioinformatics and Genomics Graduate Program, The Huck Institutes of the Life Sciences, Penn State University, University Park, Pennsylvania, United States

3 Institute for Computational and Data Sciences, Penn State University, University Park, Pennsylvania, United States

* To whom correspondence should be addressed: epo2@psu.edu 
Table S1. $\Delta \Delta G^{\ddagger}$ (kcal/mol) calculated from QM/MM umbrella sampling MD simulations at grid points on the $(\varphi, \theta)$ plane with $95 \%$ confidence intervals about the mean.

\begin{tabular}{|c|c|c|c|c|c|c|c|c|c|c|}
\hline & \multicolumn{9}{|c|}{$\theta$ (degree) } \\
\hline & & 0.0 & 22.5 & 45.0 & 67.5 & 90.0 & 112.5 & 135.0 & 157.5 & 180.0 \\
\hline \multirow{16}{*}{ 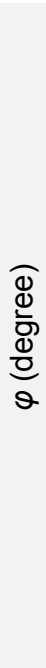 } & -180.0 & $1.64 \pm 0.94$ & & $-3.12 \pm 0.96$ & & $-0.69 \pm 0.95$ & & $-2.17 \pm 1.01$ & & $-0.44 \pm 0.97$ \\
\hline & -157.5 & & $-1.52 \pm 0.94$ & & $-0.45 \pm 0.91$ & & $-1.61 \pm 0.94$ & & $0.93 \pm 0.94$ & \\
\hline & -135.0 & & & $0.69 \pm 0.92$ & & $-3.23 \pm 0.96$ & & $-1.54 \pm 0.96$ & & \\
\hline & -112.5 & & $-1.90 \pm 0.94$ & & $0.14 \pm 0.93$ & & $-0.32 \pm 0.94$ & & $-1.33 \pm 0.92$ & \\
\hline & -90.0 & & & $-3.39 \pm 0.92$ & & $-3.72 \pm 0.90$ & & $-3.27 \pm 0.92$ & & \\
\hline & -67.5 & & $-0.31 \pm 0.98$ & & $-1.31 \pm 0.97$ & & $-0.96 \pm 0.93$ & & $0.38 \pm 0.92$ & \\
\hline & -45.0 & & & $-1.83 \pm 0.92$ & & $-2.22 \pm 0.93$ & & $-0.80 \pm 0.97$ & & \\
\hline & -22.5 & & $0.68 \pm 0.95$ & & $-1.27 \pm 1.00$ & & $-2.08 \pm 0.94$ & & $-1.29 \pm 0.95$ & \\
\hline & 0.0 & & & $-2.51 \pm 0.89$ & & $-2.00 \pm 0.92$ & & $-1.20 \pm 0.95$ & & \\
\hline & 22.5 & & $2.71 \pm 0.98$ & & $-0.96 \pm 0.91$ & & $1.65 \pm 0.96$ & & $-0.78 \pm 0.97$ & \\
\hline & 45.0 & & & $2.72 \pm 0.93$ & & $-0.67 \pm 0.93$ & & $2.41 \pm 0.97$ & & \\
\hline & 67.5 & & $-0.51 \pm 0.94$ & & $2.93 \pm 1.00$ & & $1.80 \pm 0.95$ & & $0.02 \pm 0.93$ & \\
\hline & 90.0 & & & $-0.16 \pm 0.92$ & & $1.90 \pm 0.93$ & & $-2.40 \pm 0.95$ & & \\
\hline & 112.5 & & $-0.79 \pm 0.94$ & & $2.66 \pm 0.96$ & & $1.15 \pm 0.99$ & & $0.67 \pm 0.99$ & \\
\hline & 135.0 & & & $1.57 \pm 0.95$ & & $2.29 \pm 0.99$ & & $-0.69 \pm 0.92$ & & \\
\hline & 157.5 & & $-1.60 \pm 0.95$ & & $0.20 \pm 0.93$ & & $-0.86 \pm 1.01$ & & $0.30 \pm 0.94$ & \\
\hline
\end{tabular}



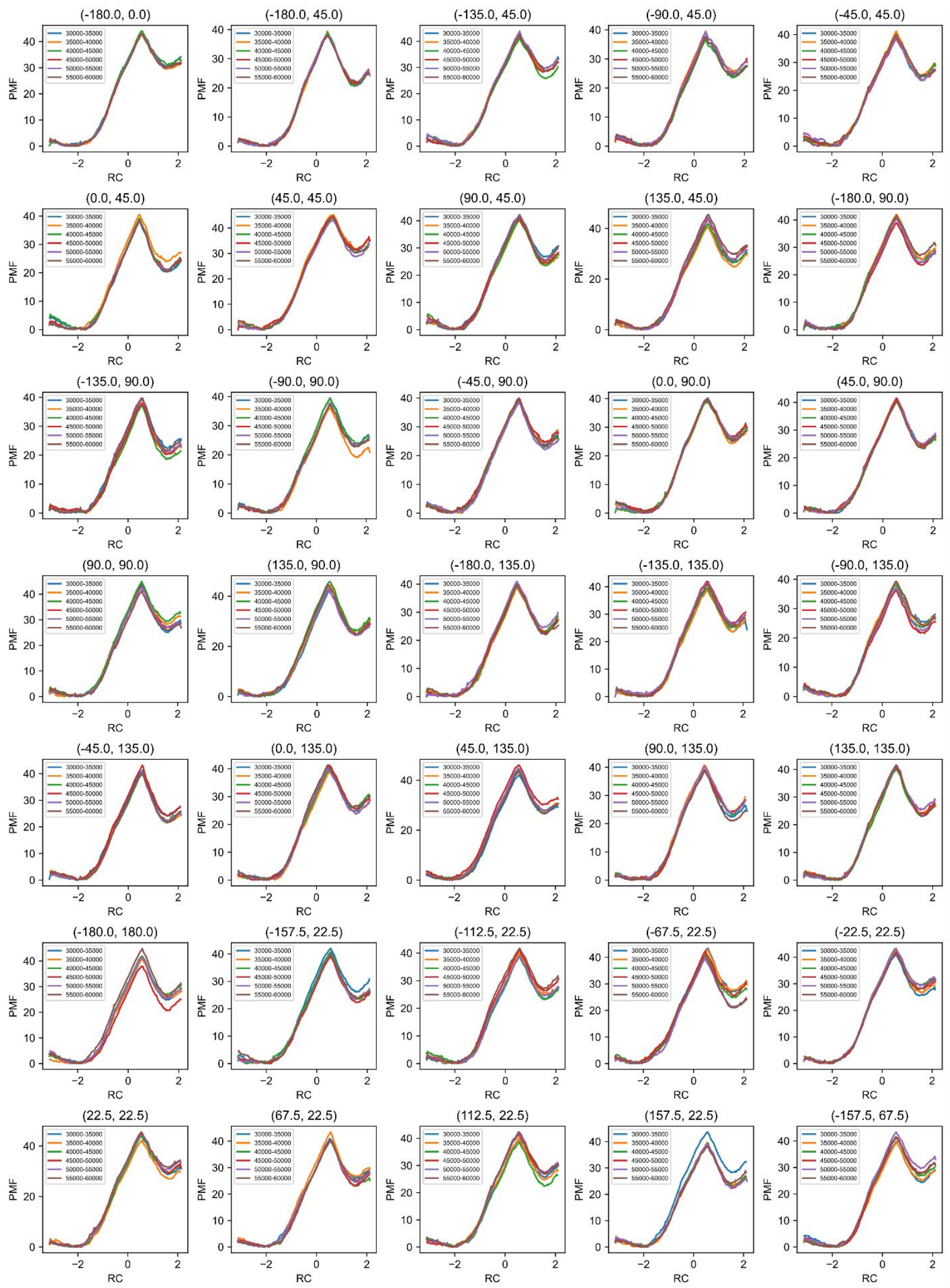

continued on the next page... 
continued from previous page...
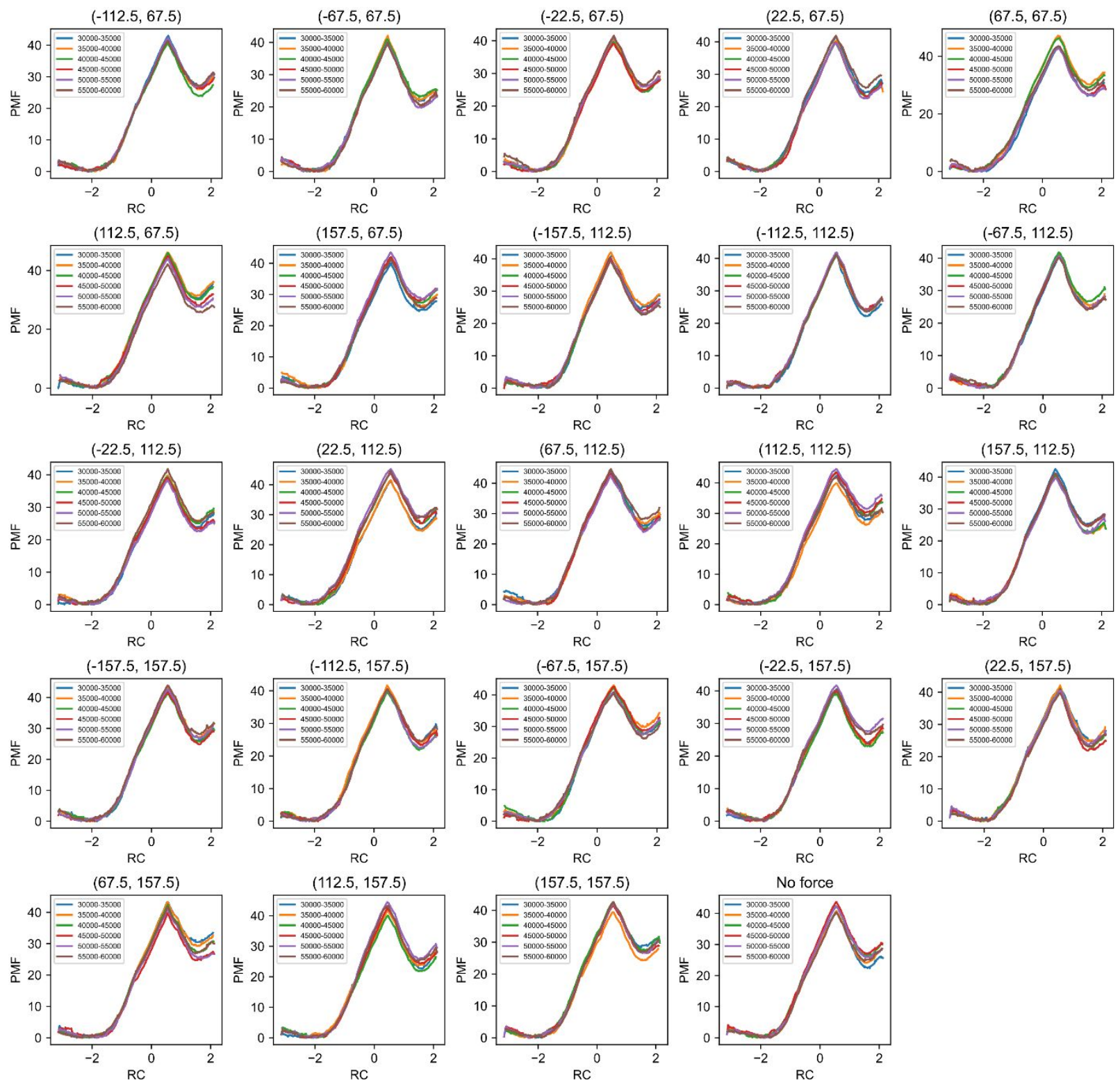

Figure S1. A test for convergence of the PMFs obtained from the last 30-ps trajectories of the QM/MM umbrella sampling simulations. The convergence was tested by calculating the PMF as a function of the reaction coordinate ('RC') obtained from 6 consecutive blocks, each 5ps in duration, of the last 30-ps of the simulation. PMFs that exhibit fluctuations around some average behavior are considered to be converged. In general most simulations are converged on the time scale of our simulations. 